1 Hacettepe Journal of Mathematics and Statistics

$\bigcap$ Volume 44 (4) (2015), 893-899

\title{
Autoisoclinism classes and autocommutativity degrees of finite groups
}

\author{
Mohammad Reza Rismanchian * and Zohreh Sepehrizadeh ${ }^{\dagger}$
}

\begin{abstract}
The notion of autoisoclinism was first introduced by Moghaddam et. al., in 2013. In this article we derive more properties of autoisoclinism and define autocommutativity degrees of finite groups. This work also generalizes some results of Lescot in 1995. Among the other results, we determine an upper bound for autocommutativity degree of finite groups.
\end{abstract}

2000 AMS Classification: 20D15, 20F28, 20P05.

Keywords: Autocommutativity degree, Autoabelian, Autoisoclinism, Absolute centre.

Received 03/02/2014 : Accepted 28/04/2014 Doi : 10.15672 /HJMS.2015449442

\section{Introduction}

If $G$ is a finite group then the commutativity degree $d(G)$ of $G$ is defined as

$$
d(G)=\frac{1}{|G|^{2}}|\{(x, y) \in G \times G \mid[x, y]=1\}|,
$$

which is the probability that two randomly chosen elements of $G$ commute, where $[x, y]=$ $x^{-1} y^{-1} x y$. The commutative degree first studied by Gustafson in 1973, where he showed that $d(G) \leq \frac{5}{8}$ for every non-abelian finite group $G$. The equality holds when $G / Z(G) \simeq$ $\mathbb{Z}_{2} \times \mathbb{Z}_{2}$. In 1995 , Lescot investigated this concept by considering the notion of isoclinism of groups. Whence he obtained certain results in this regard. In 2007, Erfanian et. al., introduced the concept of relative commutativity degree $d(H, G)$ of a subgroup $H$ in a given group $G$ as

$$
d(H, G)=\frac{1}{|H||G|}|\{(x, y) \in H \times G \mid[x, y]=1\}|,
$$

\footnotetext{
${ }^{*}$ Department of Pure Mathematics, Shahrekord University, Shahrekord, Iran. Corresponding author.

Email: rismanchian@sci.sku.ac.ir

${ }^{\dagger}$ Department of Pure Mathematics, Shahrekord University, Shahrekord, Iran.

Email: zohreh.sepehri@gmail.com
} 
which is the probability that an arbitrary element of $H$ commutes with an element of $G$. In this article, we introduce the autocommutativity degree and the relative autocommutativity degree of a subgroup $H$ of $G$, denoted by $d_{\text {aut }}(G)$ and $d_{\text {aut }}(H, G)$, respectively, which are defined as follows:

$$
d_{\text {aut }}(G)=\frac{1}{|G||A u t(G)|}|\{(x, \alpha) \in G \times \operatorname{Aut}(G) \mid[x, \alpha]=1\}|,
$$

and

$$
d_{\text {aut }}(H, G)=\frac{1}{|H||A u t(G)|}|\{(x, \alpha) \in H \times \operatorname{Aut}(G) \mid[x, \alpha]=1\}|,
$$

where $[x, \alpha]=x^{-1} x^{\alpha}$. Clearly, $d_{a u t}(G)=1$ if and only if $\operatorname{Aut}(G)=\{1\}$, that is, if and only if $|G| \leq 2$.

In Hegarty [5], the characteristic subgroups $K(G)$ and $L(G)$ of $G$ are defined as follows:

$$
K(G)=\langle[x, \alpha] \mid x \in G, \alpha \in \operatorname{Aut}(G)\rangle,
$$

and

$$
L(G)=\{x \mid[x, \alpha]=1, \forall \alpha \in \operatorname{Aut}(G)\},
$$

which are called autocommutator subgroup and absolute centre of $G$, respectively. One can easily check that $K(G)$ contains the derived subgruop $G^{\prime}$ of $G$ and $L(G)$ is contained in the centre, $Z(G)$, of $G$.

\section{Results on the relative autocommutativity degree}

Let $G$ be a group, and $\alpha$ be an automorphism of $G$. The $\operatorname{subgroup~} C_{G}(\alpha)$ of $G$ is defined by

$$
C_{G}(\alpha)=\{x \in G \mid[x, \alpha]=1\} .
$$

The following lemma gives an upper bound for $d_{\text {aut }}(G)$, which is similar to Lemma 1.3 of $[6]$.

2.1. Lemma. Let $G$ be a finite nontrivial group. If $p$ is the smallest prime divisor of $|G|$, then $d_{\text {aut }}(G) \leq \frac{p-1}{p|\operatorname{Aut}(G)|}+\frac{1}{p}$.

Proof. Let $p$ be the smallest prime divisor of $|G|$. Then $\left|C_{G}(\alpha)\right| \leq|G| / p$ for $\alpha \neq 1$ which $\alpha \in \operatorname{Aut}(G)$ and hence

$$
\begin{array}{rlrl}
|G| \mid \text { Aut }(G) \mid d_{\text {aut }}(G) & = & \sum_{\alpha \in A u t(G)}\left|C_{G}(\alpha)\right| \\
& = & \sum_{\alpha=1}\left|C_{G}(\alpha)\right|+\sum_{\alpha \in \text { Aut }(G) \backslash\{1\}}\left|C_{G}(\alpha)\right| \\
& = & & |G|+\left|C_{G}(\alpha)\right|(\mid \text { Aut }(G) \mid-1) \\
& \leq & & |G|+\frac{|G|}{p}(\mid \text { Aut }(G) \mid-1) \\
& = & & \frac{|G|}{p}((p-1)+\mid \text { Aut }(G) \mid) .
\end{array}
$$

Therefore

$$
d_{\text {aut }}(G) \leq \frac{p-1}{p|\operatorname{Aut}(G)|}+\frac{1}{p} .
$$


2.2. Example. Consider the Klein four-group,

$$
V_{4}=\left\langle a, b \mid a^{2}=b^{2}=(a b)^{2}=1\right\rangle .
$$

It is easy to see that $\left|A u t\left(V_{4}\right)\right|=6$. Thus

$$
\begin{aligned}
d_{\text {aut }}\left(V_{4}\right) & =\frac{|\{(1, \alpha) \mid \alpha(1)=1\}|+3|\{(b, \alpha) \mid \alpha(b)=b\}|}{\left|V_{4}\right|\left|\operatorname{Aut}\left(V_{4}\right)\right|} \\
& =\frac{6+6}{24}=\frac{1}{2}<\frac{7}{12}
\end{aligned}
$$

$\left(\alpha \in \operatorname{Aut}\left(V_{4}\right)\right)$ which satisfies Lemma 2.1.

The following theorem is a generalization of Lemma 2.1 of [1]. Moreover its first part implies Lemma 2.2, Proposition 2.1 and Theorem 2.2 of [8].

2.3. Theorem. Let $G$ be a finite group and $H \leq K \leq G$. Then $d_{\text {aut }}(K, G) \leq d_{\text {aut }}(H, G)$, and equality holds if and only if $K=H C_{K}(\alpha)$ for all $\alpha \in \operatorname{Aut}(G)$.

Proof. Put $A=\left\{h \in H \mid h^{\alpha}=h\right\}$ and $B=\left\{k \in K \mid k^{\alpha}=k\right\}$. Clearly the map $\{h A \mid h \in H\} \rightarrow\{k B \mid k \in K\}$, with $h A \mapsto h B$ is one-to-one. So we have $\frac{|H|}{\left|\left\{h \mid h^{\alpha}=h\right\}\right|} \leq$ $\frac{|K|}{\left|\left\{k \mid k^{\alpha}=k\right\}\right|}$, that is $\frac{\left|C_{K}(\alpha)\right|}{|K|} \leq \frac{\left|C_{H}(\alpha)\right|}{|H|}$ for each $\alpha \in \operatorname{Aut}(G)$. Hence

$$
\begin{aligned}
d_{\text {aut }}(K, G) & =\frac{1}{|A u t(G)|} \sum_{\alpha \in \operatorname{Aut}(G)} \frac{\left|C_{K}(\alpha)\right|}{|K|} \\
& \leq \frac{1}{|A u t(G)|} \sum_{\alpha \in A u t(G)} \frac{\left|C_{H}(\alpha)\right|}{|H|} \\
& =d_{\text {aut }}(H, G) .
\end{aligned}
$$

Also $d_{\text {aut }}(K, G)=d_{\text {aut }}(H, G)$ if and only if $\frac{\left|C_{K}(\alpha)\right|}{|K|}=\frac{\left|C_{H}(\alpha)\right|}{|H|}$ for all $\alpha \in \operatorname{Aut}(G)$, which is equivalent to $K=H C_{K}(\alpha)$ for all $\alpha \in A u t(G)$.

Clearly for any subgroup $H \leq G$, we have $d_{\text {aut }}(G) \leq d_{\text {aut }}(H, G)$. For example, in the Klein four-group we have $d_{\text {aut }}\left(\bar{V}_{4}\right)=\frac{1}{2} \leq \frac{2}{3}=d_{\text {aut }}\left(<\bar{a}>, V_{4}\right)$.

2.4. Definition. Two groups $G$ and $H$ are autoisoclinic, (written $G \sim_{a u t} H$ ), if there exists isomorphisms $\alpha, \beta$ and $\gamma$, as follows:

$$
\begin{aligned}
& \alpha: \frac{G}{L(G)} \longrightarrow \frac{H}{L(H)} \\
& \beta: K(G) \longrightarrow K(H) \\
& \gamma: \operatorname{Aut}(G) \longrightarrow \operatorname{Aut}(H),
\end{aligned}
$$

where $\alpha$ induces $\beta$ in following sense: if $g \in G, h \in \alpha(g L(G))$ and if $\varphi_{H}=\gamma\left(\varphi_{G}\right)$, then $\beta\left(\left[g, \varphi_{G}\right]\right)=\left[h, \varphi_{H}\right]$.

The pair $(\alpha \times \gamma, \beta)$ is called an autoisoclinism between $G$ and $H$, see also [7] (and [6] for isoclinism).

Let $(\alpha \times \gamma, \beta)$ be an autoisoclinism between the groups $G$ and $H$, then the following diagram is commutative: 


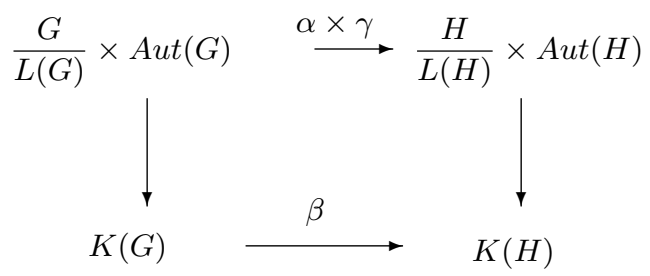

Next, we present the following lemma which is similar to Lemma 2.4 of [6].

2.5. Lemma. Let $G$ and $H$ be two autoisoclinic finite groups, then $d_{a u t}(G)=d_{a u t}(H)$.

Proof. Let pair $(\alpha \times \gamma, \beta)$ be an autoisoclinism from $G$ to $H$, then one has

$$
\begin{aligned}
\mid \frac{G}{L(G)} \| \text { Aut }(G) \mid d_{\text {aut }}(G) & =\frac{1}{|L(G)|}|G| \mid \text { Aut }(G) \mid d_{\text {aut }}(G) \\
& =\frac{1}{|L(G)|}\left|\left\{\left(g, \varphi_{G}\right) \in G \times A u t(G) \mid\left[g, \varphi_{G}\right]=1\right\}\right| \\
& =\left|\left\{\left(g L(G), \varphi_{G}\right) \in \frac{G}{L(G)} \times \operatorname{Aut}(G) \mid \beta\left(\left[g, \varphi_{G}\right]\right)=1\right\}\right| \\
& =\left|\left\{\left(h L(H), \varphi_{H}\right) \in \frac{H}{L(H)} \times A u t(H) \mid\left[h, \varphi_{H}\right]=1\right\}\right| \\
& =\frac{1}{|L(H)|}\left|\left\{\left(h, \varphi_{H}\right) \in H \times A u t(H) \mid\left[h, \varphi_{H}\right]=1\right\}\right| \\
& =\frac{1}{|L(H)|}|H \| A u t(H)| d_{\text {aut }}(H) \\
& =\left|\frac{H}{L(H)}\right||A u t(H)| d_{\text {aut }}(H) .
\end{aligned}
$$

But $\frac{G}{L(G)}$ and $\frac{H}{L(H)}$ are isomorphic, so $\left|\frac{G}{L(G)}\right|=\left|\frac{H}{L(H)}\right|$. From the fact that $A u t(G)$ and $\operatorname{Aut}(H)$ are isomorphic, we conclude $|A u t(G)|=|A u t(H)|$, from which the equality $d_{\text {aut }}(G)=d_{\text {aut }}(H)$ follows.

\section{Results on autoisoclinism}

We begin this section by establishing some elementary lemmas which will be used to derive the results on autoisoclinism (see also $[2,6]$ ). Now, we present the following lemma which is similar to Lemma 2.6 of [6].

3.1. Lemma. Let $S$ be a characteristically simple group of order more than two, then any group $G$ autoisoclinic to $S$ is isomorphic to $S \times L(G)$.

Proof. By order of $S$ we have $K(S) \neq<1>$ and $L(S) \neq S$. Suppose $G \sim_{\text {aut }} S$. Hence $K(G) \simeq K(S)$. Since $S$ is a characteristically simple group, then $K(S)=S \simeq K(G)$. Thus $K(G) \cap L(G) \subseteq L(K(G))=\{1\}$. On the other hand $\frac{G}{L(G)} \simeq \frac{S}{L(S)} \simeq S$ because $G \sim_{a u t} S$ and $S$ is a characteristically simple group. Hence

$$
\begin{aligned}
& K\left(\frac{G}{L(G)}\right) \simeq K(S)=S \simeq \frac{G}{L(G)} \\
& \Rightarrow \frac{L(G) K(G)}{L(G)}=K\left(\frac{G}{L(G)}\right)=\frac{G}{L(G)} \\
& \Rightarrow G=K(G) L(G)=K(G) \times L(G) \simeq S \times L(G) .
\end{aligned}
$$


3.2. Lemma. Let $H$ be a finite subgroup of $G$, where $H$ and $G$ are autoisoclinic, then $G=H L(G)$.

Proof. If $H$ is finite group autoisoclinic to $G$, then $\frac{G}{L(G)} \simeq \frac{H}{L(H)}$ is also finite. But

$$
\begin{aligned}
\left|\frac{G}{L(G)}\right| & \geq\left|\frac{H L(G)}{L(G)}\right| \\
& =\left|\frac{H}{H \cap L(G)}\right| \\
& =\left|\frac{H}{L(H)}\right|\left|\frac{L(H)}{H \cap L(H)}\right| \\
& \geq\left|\frac{H}{L(H)}\right| \\
& =\left|\frac{G}{L(G)}\right| .
\end{aligned}
$$

This implies that $G=H L(G)$.

3.3. Lemma. Let $H$ be a characteristic subgroup of finite group $G$, and $G=H L(G)$ such that $\operatorname{Aut}(H) \simeq \operatorname{Aut}(G)$. Then $G$ and $H$ are autoisoclinic.

Proof. If $H$ is a characteristic subgroup of $G$, then $H \cap L(G) \subseteq L(H)$. Also $L(H) \simeq$ $H \cap L(G)$ because $\operatorname{Aut}(H) \simeq \operatorname{Aut}(G)$, and

$$
\begin{aligned}
\frac{H}{L(H)} & =\frac{H}{L(G) \cap H} \\
& \simeq \frac{H L(G)}{L(G)} \\
& =\frac{G}{L(G)},
\end{aligned}
$$

the isomorphism $i_{1}: H / L(H) \longrightarrow G / L(G)$ being induced by the inclusion $i: H \longrightarrow G$. Furthermore, let $g \in G, \alpha \in A u t(G)$, then $g=l h$ for some $l \in L(G)$ and $h \in H$. Hence $[g, \alpha]=[l h, \alpha]=(l h)^{-1}(l h)^{\alpha}=h^{-1} l^{-1} l^{\alpha} h^{\alpha}=h^{-1} h^{\alpha}=[h, \alpha] \in K(H)$, On the other hand $K(H) \simeq\langle[h, \alpha] \mid h \in H, \alpha \in A u t(G)\rangle \subseteq K(G)$, and so $K(G)=K(H)$. This argument shows that $\left(i_{1} \times 1_{A u t(G)}, 1_{K(G)}\right)$ is an autoisoclinism from $H$ to $G$.

3.4. Theorem. Let $G$ be a finite group such that $G=H L(G)$ where $H$ is a characteristic subgroup of $G$ with $\operatorname{Aut}(H) \simeq \operatorname{Aut}(G)$. Then $d_{\text {aut }}(G)=d_{\text {aut }}(H)$.

Proof. It follows from Lemma 2.5 and Lemma 3.3 .

The following lemma is similar to Lemma 1.3 of [2], will be used in the next theorem.

3.5. Lemma. Let $G$ be a group with characteristic subgroup $N$. Then $G / N \sim_{\text {aut }} G /(N \cap K(G))$. In particular, if $N \cap K(G)=\{1\}$, then $G \sim_{a u t} G / N$. Conversely, if $|K(G)|<\infty$ and $G \sim_{\text {aut }} G / N$, then $N \cap K(G)=\{1\}$.

Proof. We set $\bar{G}=G / N$ and $\hat{G}=G /(N \cap K(G))$. For any $k_{1}, k_{2} \in K(G), \bar{k}_{1}=\bar{k}_{2} \Leftrightarrow$ $\hat{k}_{1}=\hat{k}_{2}$. For $g \in G$ and $\varphi \in A u t(G)$, we have therefore, $[\bar{g}, \bar{\varphi}]=\overline{1} \Leftrightarrow[\hat{g}, \hat{\varphi}]=\hat{1}$ (because $N$ is characteristic subgroup of $G)$, where $\bar{\varphi}: g N \rightarrow \varphi(g) N$ and $\hat{\varphi}: g(N \cap K(G)) \rightarrow$ $\varphi(g)(N \cap K(G))$. This implies that $\bar{g} \in L(\bar{G})$ if and only if $\hat{g} \in L(\hat{G})$. Let $\alpha(\bar{g} L(\bar{G}))=$ $\hat{g} L(\hat{G})$. Then $\alpha$ is an isomorphism of $\bar{G} / L(\bar{G})$ onto $\hat{G} / L(\hat{G})$. If $\gamma\left(\bar{\varphi}_{\bar{G}}\right)=\hat{\varphi}_{\hat{G}}$, then $\gamma$ is an isomorphism of $\operatorname{Aut}(\bar{G})$ onto $\operatorname{Aut}(\hat{G})$. Let $k \in K(G)$ and denote $\beta(\bar{k})=\hat{k}$. Then $\beta$ defines an isomorphism of $K(\bar{G})$ onto $K(\hat{G})$ and $\beta$ is induced by $\alpha$ in Definition 2.4 . 
Conversely, if $N \unlhd G$ and $G \sim_{a u t} G / N$, then

$$
K(G) \simeq K(G / N)=K(G) N / N \simeq K(G) /(N \cap K(G)) .
$$

Thus, if $|K(G)|<\infty$, then $N \cap K(G)=\{1\}$.

The following theorem follows from the above lemma and is similar to Theorem 1.4 of [2].

3.6. Theorem. Let $G$ and $H$ be finite groups. Then $G$ and $H$ are autoisoclinic if and only if there exists finite groups $C, L_{G}, L_{H}, C_{G}$ and $C_{H}$ such that $G \simeq C / L_{H}$ and $H \simeq C / L_{G}$ and the following two (equivalent) properties hold:

(i) $G \simeq C / L_{H} \sim_{\text {aut }} C \sim_{\text {aut }} C / L_{G} \simeq H$,

(ii) $C / L_{H} \times C / K(C) \sim_{\text {aut }} C_{H} \simeq C \simeq C_{G} \sim_{\text {aut }} C / L_{G} \times C / K(C)$, where $C_{H}$ and $C_{G}$ are subgroups of $C / L_{H} \times C / K(C)$ and $C / L_{G} \times C / K(C)$ respectively.

Proof. One part of the theorem is trivial. Assume that $G \sim_{a u t} H$, and let $\beta$ be the isomorphism between $K(G)$ and $K(H)$ given in Definition 2.4. Finally, let $C$ be the direct product of $G$ and $H$ with identified factor groups $G / L(G)$ and $H / L(H)$. If

$$
L_{H}=\{(1, l) \mid l \in L(H)\} \text { and } L_{G}=\{(l, 1) \mid l \in L(G)\},
$$

then we have $C / L_{H} \simeq G$ and $C / L_{G} \simeq H$, where $L_{H} \simeq L(H)$ and $L_{G} \simeq L(G)$.

(i) It follows from Definition 2.4 that $K(C)$ is generated by elements of the form

$$
\left(\left[g, \varphi_{G}\right], \beta\left(\left[g, \varphi_{G}\right]\right)\right)
$$

where $g \in G, \varphi_{G} \in \operatorname{Aut}(G)$. We claim that

$$
K(C) \cap L_{G}=K(C) \cap L_{H}=1 .
$$

For, if $(1, l)=(g, h) \in K(C)$, then $h=\beta(g)=1$. Similarly for $K(C) \cap L_{G}$. By Lemma 3.5 we therefore have

$$
C / L_{H} \sim_{\text {aut }} C \sim_{\text {aut }} C / L_{G} .
$$

(ii) Let

$$
C_{G}=\left\{\left(c L_{G}, c K(C) \mid c \in C\right\} .\right.
$$

$C_{G}$ is a group, isomorphic to $C$, since $K(C) \cap L_{G}=\{1\}$. Moreover, it follows from Lemma 3.3 that $C_{G} \sim_{a u t} C / L_{G} \times C / K(C)$. Now we have the equality

$$
C_{G} L\left(C / L_{G} \times C / K(C)\right)=C / L_{G} \times C / K(C) .
$$

To see this, let $x=\left(c_{1} L_{G}, c_{2} K(C)\right)$ be an element of the direct product of the groups $C / L_{G}$ and $C / K(C)$. Then $x=y l$, where $y=\left(c_{1} L_{G}, c_{1} K(C)\right) \in$ $C_{G}$, and $l=\left(L_{G}, c_{1}{ }^{-1} c_{2} K(C)\right)$. Since $C / K(C)$ is an autoabelian group, then $L(C / K(C))=C / K(C)$ and $L_{G}$ is identity of $C / L_{G}$. It follows that $l \in$ $L\left(C / L_{G} \times C / K(C)\right)$.

Similarly

$$
C \simeq C_{H} \sim_{\text {aut }} C / L_{H} \times C / K(C)
$$

Acknowledgments. The authors are grateful to referee for the useful comments, which improved the presentation of the paper. The first author was also partially supported by the Center of Excellence for Mathematics, Shahrekord University, Iran. 


\section{References}

[1] R. Barzegar, A. Erfanian and M. Farrokhi D.G, Finite groups with three relative commutativity degrees, Bull. Iranian Math. Soc., 39(2) (2013) 271-280.

[2] J. C. Bioch, On n-isoclinic groups, Indag. Math., 79 (1976) 400-407.

[3] A. Erfanian, R. Rezaei and P. Lescot, On the relative commutativity degree of a subgroup of a finite group, Comm. Algebra, 35(12) (2007) 4183-4197.

[4] W. H. Gustafson, What is the probability that two group elements commute ?, Amer. Math. Monthly, 80 (1973) 1031-1034.

[5] P. V. Hegarty, The absolute centre of a group, J. Algebra, 169(3) (1994) 929-935.

[6] P. Lescot, Isoclinism classes and commutativity degrees of finite groups, J. Algebra, 177 (1995) 847-869.

[7] M. R. R. Moghaddam, M. J. Sadeghifard and M. Eshrati, Some properties of autoisoclinism of groups, Fifth International group theory conference, Islamic Azad university, MashhadIran, 13-15 March 2013.

[8] M. R. R. Moghaddam, F. Saeedi and E. Khamseh, The probability of an automorphism fixing a subgroup element of a finite group, Asian-Eur. J. Math., 4(2) (2011) 301-308. 
